certain sorts of societies. A scientist wants to know why, but for the politician and the economist this limits courses of action. There will, of course, be alternatives available, but in Wilson's world they are appreciably more restricted than is generally thought. Economists do not appreciate the options because they have yet to think of them.

Wilson is a kind man, but he has no problems producing withering denouncements where they are called for. His description of our ecological plight, and his heartfelt plea for understanding and urgent action, are written with such authority, clarity and passion that I have read nothing to touch them. Nobel laureates in economics who fail to appreciate the world's finite resources and who argue for our wants in the short term rather than our needs for a sustained future are targets in this book. Evolutionary psychologists also get a timely warning. I was delighted to see one of their gurus pointing out so very dispassionately that, although Darwinian explanations abound, the theory is in its infancy and the database against which ideas are being tested remains poor.

Within this second theme, Wilson is at pains to circumscribe his own limits. Returning to art, he writes: "While biology has an important part to play in scholarly criticism, the creative arts themselves can never be locked in by this or any other discipline of science.... Works of art communicate feeling directly from mind to mind, with no intent to explain why the impact occurs. In this defining quality, the arts are the antithesis of science."

Wilson has had such a broad agenda through his career that he has frequently sought the advice of others for complementary expertise. For some of his research monographs he has collaborated with coauthors. At one end of the axis of excellence was Robert MacArthur. Fortunately, like George Oster, most of Wilson's collaborators lie close to MacArthur in this respect. And as with Sociobiology, so with Consilience - the vision is Wilson's but much of the detailed understanding of mechanism is necessarily gleaned from experts in their own fields. The responses from those working in the areas to which the clarion calls are directed may depend on how well Wilson has chosen his examples, and how accurately he has described the state of their art.

His own pool of knowledge and grasp of contemporary advances is astonishing, but even so we can expect the nitpickers to gather. I hope they will take time to evaluate the broader canvas and, where necessary, supply their own case studies in mounting whatever responses this monumental work demands. In any event, it is time to get on with the job. If we have to go through a bruising session like that following Sociobiology, I think that this time most evolutionary biologists will stand behind Wilson, albeit obliquely. He deserves no less.

Some will argue that to bring evolutionary biology and the associated epigenetic rules into such areas as art appreciation will turn us into Philistines. They should be directed to Darwin's response to the evolutionary interpretation for life itself in the last paragraph of Origin of Species. The grandeur in his view of life provided us with a new dimension for appreciating the natural world but removed none of the old. Or, as Wilson might have it, our biophilia was intensified.

Paul H. Harvey is in the Department of Zoology,

University of Oxford, South Parks Road, Oxford OX1 3PS, UK.

\section{At the limit}

\section{Impossibility}

by John D. Barrow

Oxford University Press: 1998. Pp. 279.

$£ 18.99, \$ 25$

\section{John L.Casti}

To those enamoured of the belief that the human spirit knows no bounds or limitations, a tour of twentieth-century science must be a rather depressing experience. If there is any common denominator running through the scientific breakthroughs of our age it is the idea that there are limits.

Starting with Einstein's speed limit of the Universe in a ray of light and Heisenberg's limits on what can be measured with certainty, through Gödel's limits on what can be known by following a set of rules and on to Arrow's famous result about the impossibility of a perfect democratic society, the history of modern science is riddled with logical and physical limits of all sorts.

In this illuminating, well-written account of Limits (with a capital L), John D. Barrow chronicles and explains the limits of science as a reality-generation mechanism - and why it matters.

As he carefully points out, limits to the ability of science to answer a given question about the world as we see it come in all sizes and shapes. There are political and ethical limits on how much latitude a society is willing to give to investigators to answer a question. There are practical limits on the amount of time or energy or money that can be spent in answering a question. And, finally, there are logical limits.

Although Barrow pays more than lip service to the former sorts of limitations on our ability to tease out the 'scheme of things', it's clear that his real focus is on logical limits. Rightly so, too, because ultimately these are the limits that count - at least for the scientist and scholar, if not the politician and social agitator. When one is looking for a needle in a haystack, it's of more than passing interest to know that a needle is really there to be found.

As Barrow points out, all the scientific knowledge we have about nature comes from models that we create of natural phenomena. These models, in turn, are almost always mathematical in character. It follows then that it's only a small approximation to say that the issue of logical limits to science comes down to the limits of computation, as any mathematical model can be regarded as an algorithm for processing inputs (the statement of the question and its circumstances) into an output (the answer).

This notion of scientific knowledge raises several fundamental questions. How does the mathematical model relate to the realworld phenomenon it purports to represent? Does the Turing-machine model of computation impose intrinsic limits on what we can know? What is the relationship between the computational powers of the human brain and those of our computing machines?

One question missing from the list and the book - arises from the assumption that our models of nature must necessarily be mathematical. This question is: is there an alternative to a mathematical formulation of a model of natural phenomena?

The difficulty here is that one is left perched on the horns of a dilemma. Either you use the mathematical representation of the question of concern, and then try to justify why mathematical insolubility implies the same for the model's real-world correlate. Or you forsake mathematics altogether, and then face the difficulty of trying to create a convincing real-world substitute for the mathematical notion of proof, in order to produce a knockdown argument for why the question of concern is logically unanswerable. Impossibility evades this dilemma simply by ignoring the non-mathematical alternative.

But no matter. Taken on a whirlwind tour of the mind, Gödel's theorem, quantum theory, free will, voting paradoxes, time travel, computational intractability, sandpile models, the topology of space, nanotechnology, the forces of nature, the evolution of the Universe, complexity science, computer chessplaying, percolation theory, human consciousness, economic forecasting, black holes, the Brouwer fixed-point theorem, artificial intelligence and the Heisenberg uncertainty principle, one can only wonder how Barrow can possibly make all these things fit together into a coherent story about the limits to science. Well, contrary to all expectations, he does make them fit and in only 250 pages!

So for about as good an account as you're going to get of where science stops, read this book. It won't tell you any final answer. But the journey is far more interesting - and important - than the destination.

John L. Casti is at the Santa Fe Institute, 1399 Hyde Park Road, Santa Fe, New Mexico 87501, USA. 